\title{
ANALYSIS ON A MODIFIED LESLIE-GOWER AND HOLLING-TYPE II PREDATOR-PREY SYSTEM INCORPORATING A PREY REFUGE AND TIME DELAY
}

\author{
SHILIANG CHEN ${ }^{1}$, WEIDE LI ${ }^{2}$, AND ZHIHUI MA ${ }^{3}$ \\ ${ }^{1,2,3}$ School of Mathematics and Statitics \\ Lanzhou University \\ Lanzhou, Gansu, 730000, P.R. CHINA
}

\begin{abstract}
This paper describes a delayed predator-prey model with modified Leslie-Grower scheme, in which the time delays are regarded as bifurcation parameters. The functional response is considered to be of Holling-type II and incorporates a constant proportional prey refuge. The model is considered from the point of view of persistence and stability for this particular functional response. The asymptotic stability of coexist equilibrium is examined by using constructed Lyapunov function. The delayed system is analyzed with focusing on the gestation delay of predator $\tau$. We investigate the occurrence of Hopf bifurcation in the neighborhood of the positive interior equilibrium. Moreover, the direction of the Hopf bifurcation and the stability of bifurcating periodic solution are analyzed. Finally, numerical simulations are carried out to verify the theoretical results of the paper.
\end{abstract}

AMS Subject Classification: $92 \mathrm{D} 25$

Key Words: time delay, prey refuge, Hopf bifurcation, limit cycle, stability, LeslieGower

Received: January 9, 2018; Accepted: March 17, 2018;

Published: April 11, 2018 doi: 10.12732/dsa.v27i2.12

Dynamic Publishers, Inc., Acad. Publishers, Ltd. https://acadsol.eu/dsa

\section{INTRODUCTION}

The dynamic relationship between prey and predator has long been and will continue to be one of the dominant themes in both ecology and mathematics ecology due to its 
universal existence and importance [4]. We concerned most is the dynamic characters caused by the interactions between different species such as the prey-predator system. The predation is the most popular research field which has been intensively studied for several decades since Lotka and Volterra [14]. Predation is a mode of life in which food is primarily obtained by killing and consuming organism, and we use functional response to describe the effect of predation. Functional response refers to the change per unit of time in the density of hosts attacked per parasite as the host density change [7]. There have been extensive studies and applications about several different types of functional response which are used in the prey-predator system to describe the stability and other dynamics, such as: Holling, Beddington-DeAngelis, CrowleyMartin, Leslie-Gower and square root type (see $[11,23,1,2,5]$ ).

Leslie introduced a prey-predator model based on the assumption that the predator population increases in a logistic form with food supply in abundance and carrying capacity of the predator in environment is proportional to the number of prey $[17,18]$. This consideration leads to

$$
\frac{d x}{d t}=\left(a_{1}-b x-q(x) y\right) x, \quad \frac{d y}{d t}=\left(a_{2}-c_{2} \frac{y}{x}\right) y .
$$

Where $x, y$, represent the population densities of prey and predator at time $t$, respectively; $a_{1}\left(a_{2}\right)$ is the intrinsic growth rate of prey(predator); $b_{1}$ describes the competition among individuals of prey; The function $q(x)$ is the so-called functional response shows the reduction in prey population due to the effect of predation; And here predator species is considered to be logistic growth with carrying capacity of $\frac{a_{2}}{c}$. In the case of prey severe scarcity, predator can switch over to other populations but its growth rate will be limited by the fact that its most favourite food is not available in abundance. Due to this consideration, Aziz-Alaoui and Okiye [3] established a modified Leslie-Gower model with the assumption of Holling type II functional response:

$$
\left\{\begin{array}{l}
\dot{x}=x\left(a_{1}-b x-\frac{c_{1} y}{x+k_{1}}\right), \\
\dot{y}=y\left(a_{2}-\frac{c_{2} y}{x+k_{2}}\right) .
\end{array}\right.
$$

Where $x, y, a_{1}, a_{2}, b, c_{2}$ have the same meaning with the classic Leslie-Gower type mentioned above. $k_{1}$ is the average saturation rate of Holling type II functional response. $c_{1}$ indicates the quality of the providing prey to predator as its favourite food. For predator can switch to other food supply, we add a positive constant $k_{2}$ to the denominator. Leslie-Gower model originally suggested by the results of analysis to the data for growth of Paramecium given by Gause [8], and till now, numerous representations of interactions between prey-predator species exemplify this model, see $[9,22]$.

In the field of ecology, the effort of prey refuge plays an important role in interaction between the prey and predator. We use the refuge to describe the phenomenon 
that some prey can escape from predation by hiding in some places where predators can not enter. The effect of refuge has been studied a lot for several decades. Liu and Han [20] studied a predator-prey diffusion model incorporating a constant prey refuge and showed the stability of the positive equilibrium and the existence of local Hopf bifurcation. Jana et al. [12] proposed an original work to describe the effort of refuge region which has a carrying capacity. They divide the environment into refuge region and predatory region and two regions have their own prey growth rate and carrying capacity respectively and there is a migration between these two regions. They analyzed the dynamic behavior of the system and shown the occurrence of Hopf bifurcation. In our work, we introduce a constant proportion refuge in which $m x$ of the prey is protected, where $m \in[0,1)$ is a constant. This leaves $(1-m) x$ of prey available to the predator.

In addition, time delay is a very important tool for studing models of population interactions, which leads to a more realistic approach to the understanding of prey-predator dynamics. More realistic models should include some of the past states of these system. A real system should be modeled by differential equations with time delays [15]. Time lag exhibit much more complicated dynamics because ordinary differential equations with a delay may cause a stable equilibrium to unstable. Prey-predator system with time delay has been studied by many authors. Nindjin et al. [21] introduced a modified Leslie-Gower model with time delay and investigated the boundedness of the solutions. Then, the global asymptotical stability of interior equilibrium was proved by using Lyapunov function method. Kar and Ghorai [13] studied a delayed modified version of Leslie-Gower and Holling type II scheme with a constant proportion harvest on both prey and predator. They investigated the influence of harvesting, the direction and the periodic solutions of Hopf bifurcation of the model by using normal form method and center manifold theorem. Yuan et al. [24] considered a modified Leslie model with time delay and Michaelis-Menten type harvesting on prey. They discussed the Hopf bifurcations at positive equibrium and its unfolding with parameter perturbations. The condition for sustainable harvesting also provided in the paper which ensures human benefit without destroys the balance of prey-predator system. Youngkun Li and Changzhao Li [19] investigated a delayed Leslie prey-predator system with Holling type I functional response incorporating a prey refuge, where time delays are regarded in the negative feedback of predator's density. They discussed the existence of global Hopf bifurcation to the system. Through the previous discussion, we introduce a single time delay $\tau$ in the negative feedback of the predator's density and take it into system (1.1) incorporating a prey refuge $m$. Hence, the model system we investigated finally becomes: 


$$
\left\{\begin{array}{l}
\dot{x}=x\left(a_{1}-b x-\frac{(1-m) c_{1} y}{(1-m) x+k_{1}}\right), \\
\dot{y}=y\left(a_{2}-\frac{c_{2} y(t-\tau)}{(1-m) x(t-\tau)+k_{2}}\right) .
\end{array}\right.
$$

The paper is organised as follows. In Section 2, we consider the boundedness of the system presented above and the permanence of the prey and predator populations. Then, we calculate the equilibria of the model with its existence conditions and determine the properties for these equilibria, especially about global stability of coexistence equilibrium. In Section 3, the delayed system is used to study the direction and stability of the Hopf bifurcation which is occurred at a critical value of the time delay. Finally, in Section 4 we validate our analytical findings by perform some numerical simulations and Section 5 gives the ecological significance of our study.

\section{STABILITY OF THE POSITIVE EQUILIBRIUM AND DYNAMIC BEHAVIOUR WHEN DELAY $\tau=0$}

\subsection{Boundedness and permanence of the system}

The initial conditions for the system (1.2) are

$$
(x(t), y(t)) \in \mathbf{C}_{+}=\mathbf{C}\left([-\tau, 0], \mathbf{R}_{+}^{\mathbf{2}}\right), x(0) \geq 0, y(0) \geq 0 .
$$

To analyze the system (1.2) we need to discuss the boundedness firstly.

Lemma 2.1. The positive quadrant int $\left(\mathbf{R}_{+}^{2}\right)$ is invariant for system (1.2).

Proof. We need to prove that for all $t \in[0, M], x(t)>0$ and $y(t)>0$, where $M$ is a positive real number. Now we use contradiction method to prove. Suppose that is not true, thus, there must exist one $t_{M}$ where $0<t<t_{M}$, such that for all $t \in\left[0, t_{M}\right]$, $x(t)>0$ and $y(t)>0$, and $x\left(t_{M}\right)=0$ or $y\left(t_{M}\right)=0$ or either. Then, we have

$$
\begin{aligned}
& x(t)=x(0) \exp \left(\int_{0}^{t} a_{1}-b x(s)-\frac{(1-m) c_{1} y(s)}{(1-m) x(s)+k_{1}} d s\right), \\
& y(t)=y(0) \exp \left(\int_{0}^{t} a_{2}-\frac{c_{2} y(s-\tau)}{(1-m) x(s-\tau)+k_{2}} d s\right) .
\end{aligned}
$$

For $(x, y)$ is defined and continuous on $\left[-\tau, t_{M}\right]$. there exist a $K \geq 0$ such that for all $t \in\left[-\tau, t_{M}\right]$,

$$
x(t)=x(0) \exp \left(\int_{0}^{t} a_{1}-b x(s)-\frac{(1-m) c_{1} y(s)}{(1-m) x(s)+k_{1}} d s\right) \geq x(0) \exp \left(-t_{M} K\right),
$$


and

$$
y(t)=y(0) \exp \left(\int_{0}^{t} a_{2}-\frac{c_{2} y(s-\tau)}{(1-m) x(s-\tau)+k_{2}} d s\right) \geq y(0) \exp \left(-t_{M} K\right) .
$$

Taking the limit, as $t \rightarrow t_{M}$, we obtain

$$
\begin{aligned}
& x\left(t_{M}\right) \geq x(0) \exp \left(-t_{M} K\right), \\
& y\left(t_{M}\right) \geq y(0) \exp \left(-t_{M} K\right) .
\end{aligned}
$$

Which contradicts the fact that at least one of $x\left(t_{M}\right), y\left(t_{M}\right)$ equals to 0 . Hence we have for all $t \in[0, M], x(t)>0, y(t)>0$.

Now we discuss about the boundedness of the system and then we shall analyze the persistence and permance of the model.

Definition 2.2. (Persistence)The system (1.1) and (1.2) is said to be weakly persistent if every solution $(x(t), y(t))$ satisfies two conditions:

$$
\begin{aligned}
& x(t) \geq 0, y(t) \geq 0, \forall t \geq 0, \\
& \lim _{t \rightarrow \infty} \sup x(t)>0, \lim _{t \rightarrow \infty} \sup y(t)>0 .
\end{aligned}
$$

system is said to be strongly persistent if every solution $(x(t), y(t))$ satisfies the following condition along with the first condition of the weak persistence.

$$
\lim _{t \rightarrow \infty} \inf x(t)>0, \lim _{t \rightarrow \infty} \inf y(t)>0 .
$$

Definition 2.3. (Permanence)The system is said to be permanent if there exist positive constant $m$ and $M$ with $0<m<M$, such that

$$
\begin{aligned}
& \min \left\{\lim _{t \rightarrow \infty} \inf x(t), \lim _{t \rightarrow \infty} \inf y(t)\right\} \geq m, \\
& \max \left\{\lim _{t \rightarrow \infty} \sup x(t), \lim _{t \rightarrow \infty} \sup y(t)\right\} \leq M .
\end{aligned}
$$

Lemma 2.4. From system (1.2) we have

$$
\lim _{t \rightarrow+\infty} \sup x(t) \leq K, \lim _{t \rightarrow+\infty} \sup y(t) \leq L,
$$

where $K=a_{1} / b$ and $L=a_{2} / c_{2}\left((1-m) K+k_{2}\right) e^{a_{2} \tau}$.

Proof. At beginning we show the following conclusion that: if $p>0, q>0$ and $d u / d t \leq(\geq) u(t)(q-p u(t)), u\left(t_{0}>0\right)$, then, $\lim _{t \rightarrow \infty} \sup u(t) \leq \frac{q}{p}\left(\lim _{t \rightarrow \infty} \inf u(t) \geq \frac{q}{p}\right)$, the proof can be found in [6]. Now from the first equation of the system we have

$$
\frac{d x}{d t}<x(t)\left(a_{1}-b x(t)\right)
$$


using the conclusion above we obtain

$$
\limsup _{t \rightarrow+\infty} x(t) \leq \frac{a_{1}}{b}=K .
$$

From the second equation we have

$$
\frac{d y}{d t}<a_{2} y(t)
$$

and for $t>\tau$ we obtain $y(t) \leq y(t-\tau) e^{a_{2} \tau}$, hence

$$
y(t-\tau) \geq y(t) e^{-a_{2} \tau} .
$$

Then, for any $\rho>1$, there exist a positive sufficiently big number $T$ such that for $t>T, x(t)<\rho K$. Then, from inequations above, we obtain for $t>T+\tau$,

$$
\frac{d y}{d t}<y(t)\left(a_{2}-\frac{c_{2} e^{-a_{2} \tau}}{\rho(1-m) K+k_{2}} y(t)\right)
$$

using the same arguments before

$$
\limsup _{t \rightarrow+\infty} y(t) \leq \frac{a_{2}}{c_{2}\left(\rho(1-m) K+k_{2}\right) e^{a_{2} \tau}}=L_{\rho} .
$$

Hence, let $\rho \rightarrow 1$ we easily get

$$
\limsup _{t \rightarrow+\infty} y(t) \leq \frac{a_{2}}{c_{2}\left((1-m) K+k_{2}\right) e^{a_{2} \tau}}=L .
$$

Lemma 2.5. The system (1.2) is permanent if it satisfies the following condition:

$$
L<\frac{a_{1} k_{1}}{c_{1}(1-m)} .
$$

Proof. From the discussion in lemma 2.4. we can choose $M=\max \{K, L\}>0$ such that

$$
\max \left\{\limsup _{t \rightarrow+\infty} x(t), \limsup _{t \rightarrow+\infty} y(t)\right\} \leq M
$$

Now to show the system is permanent we only need to find a positive number $m>0$, such that

$$
\min \left\{\liminf _{t \rightarrow+\infty} x(t), \liminf _{t \rightarrow+\infty} y(t)\right\} \geq m
$$

from the prey equation of our system, and for time $t$ large enough, we have $y(t)<\rho L$, where $\rho>1$. Then

$$
\frac{d y}{d x}>x\left(a_{1}-b x-\frac{c_{1}(1-m) \rho L}{k_{1}}\right)
$$


Thus, letting $\rho \rightarrow 1$ and by using the conclusion before we obtain

$$
\liminf _{t \rightarrow+\infty} x(t) \geq \frac{1}{b}\left(a_{1}-\frac{c_{1}(1-m) L}{k_{1}}\right) .
$$

Then we denote that $P=\frac{1}{b}\left(a_{1}-\frac{c_{1}(1-m) L}{k_{1}}\right)$. and we need $P>0$, thus $L<\frac{a_{1} k_{1}}{c_{1}(1-m)}$.

On the other hand, for predator population $y$, there must have a sufficient big constant $T_{\rho}$, for $t>T_{\rho}, x(t)>P / \rho$ and $y(t)<\rho L$, where $\rho>1$.

Then we consider $t>T_{\rho}+\tau$,

$$
\frac{d y}{d t}>y(t)\left(a_{2}-\frac{\rho c_{2}}{(1-m) P+\rho k_{2}} y(t-\tau)\right)
$$

from $y(t)<\rho L$ we have

$$
\frac{d y}{d t}>-\frac{\rho^{2} c_{2} L}{(1-m) P+\rho k_{2}} y(t)
$$

for $t>T_{\rho}+\tau$ we also have

$$
y(t-\tau)<y(t) \exp \left(\frac{\rho^{2} c_{2} L \tau}{(1-m) P+\rho k_{2}}\right) .
$$

Then, above discussion lead to, for $t>T_{\rho}+\tau$

$$
\frac{d y}{d t}>y(t)\left(a_{2}-\frac{\rho c_{2}}{(1-m) P+\rho k_{2}} \exp \left(\frac{\rho^{2} c_{2} L \tau}{(1-m) P+\rho k_{2}}\right) y(t)\right) .
$$

Hence,

$$
\liminf _{t \rightarrow+\infty} y(t) \geq \frac{a_{2}\left((1-m) P+\rho k_{2}\right)}{\rho c_{2}} \exp \left(-\frac{\rho^{2} c_{2} L \tau}{(1-m) P+\rho k_{2}}\right),
$$

letting $\rho \rightarrow 1$ we get

$$
\liminf _{t \rightarrow+\infty} y(t) \geq \frac{a_{2}\left((1-m) P+k_{2}\right)}{c_{2}} \exp \left(-\frac{c_{2} L \tau}{(1-m) P+k_{2}}\right)=P^{\prime} .
$$

Thus, let $m=\min \left\{P, P^{\prime}\right\}>0$, we have proved the system is permanent.

\subsection{Equilibrium and stability analysis}

In this section, to investigate the non-delay system first, we rewrite the model system into the form of

$$
\left\{\begin{array}{l}
\dot{x}=x\left(a_{1}-b x-\frac{(1-m) c_{1} y}{(1-m) x+k_{1}}\right), \\
\dot{y}=y\left(a_{2}-\frac{c_{2} y}{(1-m) x+k_{2}}\right) .
\end{array}\right.
$$


by setting $\dot{x}=\dot{y}=0$ we get the equilibriums of system (1.2) which are independent of the time delay $\tau$.

1) The trivial equilibrium $E_{0}=(0,0)$;

2) The boundary equilibrium $E_{1}=\left(a_{1} / b, 0\right)$.

The boundary equilibrium $E_{2}=\left(0, a_{2} k_{2} / c_{2}\right)$;

3) The interior equilibrium $E^{*}=\left(x^{*}, y^{*}\right)$ exist if $m>1-\frac{a_{1} c_{2} k_{1}}{a_{2} c_{1} k_{2}}$.

To verify the interior equilibrium we need to find the positive solution of the following equations.

$$
\left\{\begin{aligned}
a_{1}-b x^{*}-\frac{c_{1}(1-m) y^{*}}{(1-m) x^{*}+k_{1}} & =0, \\
a_{2}-\frac{c_{2} y^{*}}{(1-m) x^{*}+k_{2}} & =0 .
\end{aligned}\right.
$$

Then we substitute $y^{*}=\left[(1-m) x^{*}+k_{2}\right] \frac{a_{2}}{c_{2}}$ into the first equation and obtain

$$
A x^{*^{2}}+B x^{*}+C=0
$$

where

$$
A=(1-m) b, \quad B=b k_{1}-(1-m)\left[a_{1}-\frac{(1-m) c_{1} a_{2}}{c_{2}}\right], \quad C=\frac{c_{1}(1-m) a_{2} k_{2}}{c_{2}}-a_{1} k_{1} .
$$

Thus

$$
x_{ \pm}^{*}=\frac{-B \pm \sqrt{B^{2}-4 A C}}{2 A} .
$$

Then we need discriminant $\triangle=B^{2}-4 A C>0$, that is

$$
\left\{b k_{1}-(1-m)\left[a_{1}-\frac{(1-m) c_{1} a_{2}}{c_{2}}\right]\right\}^{2}+4(1-m) b\left(\frac{c_{1}(1-m) a_{2} k_{2}}{c_{2}}-a_{1} k_{1}\right)>0,
$$

which leads to $C=\frac{c_{1}(1-m) a_{2} k_{2}}{c_{2}}-a_{1} k_{1}$ should be positive. Hence,

$$
m>1-\frac{a_{1} c_{2} k_{1}}{a_{2} c_{1} k_{2}}
$$

and the positive solution of $(2.1)$ should be $x^{*}=\frac{-B+\sqrt{B^{2}-4 A C}}{2 A}$ and $y^{*}=\frac{a_{2}}{c_{2}}[(1-$ $\left.m) x^{*}+k_{2}\right]$.

We deal with the local stability of each equilibrium point by computing the corresponding Jacobian matrix to each equilibrium. For stability of the equilibrium points, the real parts of the eigenvalues of the Jacobian matrix must be negative.

The Jacobian matrix of trivial point $E_{0}$ is given by

$$
J_{0}=\left(\begin{array}{cc}
-a_{1} & 0 \\
0 & a_{2}
\end{array}\right),
$$


and the eigenvalues are obviously $-a_{1}$ and $a_{2}$. Therefore, the system (2.1) is always unstable around $E_{0}$ which is an unstable node point.

The Jacobian matrix of the point $E_{1}=\left(\frac{a_{1}}{b}, 0\right)$ is

$$
J_{1}=\left(\begin{array}{cc}
-a_{1} & -\frac{a_{1} c_{1}(1-m)}{(1-m) a_{1}+k_{1} b} \\
0 & a_{2}
\end{array}\right) .
$$

Then $J_{1}$ has a negative eigenvalue $-a_{1}$ and a positive eigenvalue $a_{2}$. The system (2.1) is always unstable around $E_{1}$ which is, in fact, a saddle point and whose stable manifold is $\mathrm{x}$-axis and repels in y-direction.

The Jacobian matrix of the point $E_{2}=\left(0, \frac{a_{2} k_{2}}{c_{2}}\right)$ is

$$
J_{2}=\left(\begin{array}{cc}
a_{1}-\frac{a_{2} c_{1} k_{2}(1-m)}{c_{2} k_{1}} & 0 \\
\frac{a_{2}^{2}(1-m)}{c_{2}} & -a_{2}
\end{array}\right) .
$$

It has a negative eigenvalue $-a_{2}$ and the other eigenvalue is negative if $a_{1}-\frac{a_{2} c_{1} k_{2}(1-m)}{c_{2} k_{1}}$ $<0$ holds, which leads to $m<1-\frac{a_{1} c_{2} k_{1}}{a_{2} c_{1} k_{2}}$. Hence $E_{2}$ is an asymptotically stable point(under $m=1-\frac{a_{1} c_{2} k_{1}}{a_{2} c_{1} k_{2}}, E_{2}$ will be stable but not asymptotically) and in the case there is no coexistence equilibrium $E^{*}$ of system (2.1). In the case of interior coexistence equilibrium exist, $E_{2}$ will be also an unstable point which is, in fact, a saddle point and whose stable manifold is y-axis.

Theorem 2.6. The dynamic system (2.1) has $E^{*}=\left(x^{*}, y^{*}\right)$ as locally asymptotically stable if $m>1-\frac{b k_{1}}{a_{1}}$.

Proof. The variational matrix of the model $(2.1)$ at the positive equilibrium $E^{*}$ is

$$
J^{*}=\left(\begin{array}{cc}
a_{1}-2 b x^{*}-\frac{c_{1} k_{1}(1-m) y^{*}}{\left[(1-m) x^{*}+k_{1}\right]^{2}} & -\frac{c_{1}(1-m) x^{*}}{(1-m) x^{*}+k_{1}} \\
\frac{c_{2} y^{*^{2}}(1-m)}{\left[(1-m) x^{*}+k_{2}\right]^{2}} & a_{2}-\frac{2 c_{2} y^{*}}{(1-m) x^{*}+k_{2}}
\end{array}\right) .
$$

It is obvious that $J_{12}<0, J_{21}>0$ and $J_{22}<0$. And the characteristic polynomial for $J^{*}$ is $\lambda^{2}-\operatorname{tr}\left(J^{*}\right) \lambda+\operatorname{det}\left(J^{*}\right)$ where $\operatorname{tr}\left(J^{*}\right)$ stands for trace and $\operatorname{det}\left(J^{*}\right)$ stands for determinant. From Routh-Hurwitz criteria, the eigenvalue $\lambda$ will have negative real parts if $\operatorname{tr}\left(J^{*}\right)<0$ and $\operatorname{det}\left(J^{*}\right)>0$. That leads to $J_{11}<0$.

From discussion above we have

$$
a_{1}-2 b x^{*}-\frac{c_{1} k_{1}(1-m) y^{*}}{\left[(1-m) x^{*}+k_{1}\right]^{2}}<0,
$$

furthermore, from the first equation of equilibrium equations (2.1) we get

$$
a_{1}-b x^{*}=\frac{c_{1} k_{1}(1-m) y^{*}}{(1-m) x^{*}+k_{1}} .
$$


Substitute (2.4) into the inequality (2.3), then

$$
a_{1}-2 b x^{*}-\frac{k_{1}\left(a_{1}-b x^{*}\right)}{(1-m) x^{*}+k_{1}}<0,
$$

which leads to

$$
-2 b(1-m) x^{*^{2}}+\left(a_{1}(1-m)-b k_{1}\right) x^{*}<0 .
$$

Thus

$$
x^{*}>\frac{a_{1}(1-m)-b k_{1}}{2 b(1-m)} .
$$

For $x^{*}$ is a positive constant, the inequality (2.5) needs to be always hold, Then, the right hand side of the inequality (2.5) must be negative:

$$
\frac{a_{1}(1-m)-b k_{1}}{2 b(1-m)}<0
$$

Hence, we obtain the condition that

$$
m>1-\frac{b k_{1}}{a_{1}}
$$

Now we have the following theorem to examine the system is globally asymptotically stable without time delay at the coexist equilibrium.

Theorem 2.7. The interior equilibrium $E^{*}$ is globally asymptotically stable if

$$
m>1-\frac{b k_{1}}{a_{1}-b x^{*}} .
$$

Proof. We constructing a suitable Lyapunov functional to prove the theorem. The function is defined as

$$
V(x, y)=V_{1}(x, y)+\mu V_{2}(x, y)
$$

where $V_{1}(x, y)=x-x^{*}-x^{*} \ln \left(\frac{x}{x^{*}}\right), V_{2}(x, y)=y-y^{*}-y^{*} \ln \left(\frac{y}{y^{*}}\right)$, the constant $\mu$ is positive and will be defined in the discussion below. This function is well defined and continuous on $\operatorname{Int}\left(\mathbb{R}_{+}^{2}\right)$. It is obvious that the function $V(x, y)$ is zero at equilibrium $E^{*}$ and is positive for all other $(x, y)$ except $E^{*}$.

The time derivative of $V(x, y)$ is given by

$$
\begin{aligned}
\frac{d V}{d t} & =\left(x-x^{*}\right)\left(a_{1}-b x-\frac{c_{1}(1-m) y}{(1-m) x+k_{1}}\right)+\mu\left(y-y^{*}\right)\left(\frac{c_{2} y}{(1-m) x+k_{2}}\right) \\
& =\left(x-x^{*}\right)\left(-b\left(x-x^{*}\right)+\frac{c_{1}(1-m) y^{*}}{(1-m) x^{*}+k_{1}}+\frac{c_{1}(1-m) y}{(1-m) x+k_{1}}\right)
\end{aligned}
$$




$$
\begin{aligned}
& +\mu\left(y-y^{*}\right)\left(\frac{c_{2} y^{*}}{(1-m) x^{*}+k_{2}}+\frac{c_{2} y}{(1-m) x+k_{2}}\right) \\
& =\left(-b+\frac{c_{1}(1-m)^{2} y^{*}}{\left((1-m) x^{*}+k_{1}\right)\left((1-m) x+k_{1}\right)}\right)\left(x-x^{*}\right)^{2} \\
& -\left(\frac{k_{2} c_{2}+(1-m) c_{2} x^{*}}{\left((1-m) x^{*}+k_{2}\right)\left((1-m) x+k_{2}\right)}\right) \mu\left(y-y^{*}\right)^{2} \\
& +\left(\frac{-\left(c_{1} k_{1}(1-m)+c_{1}(1-m)^{2} x^{*}\right)}{\left((1-m) x^{*}+k_{1}\right)\left((1-m) x+k_{1}\right)}+\frac{\mu(1-m) c_{2} y^{*}}{\left((1-m) x^{*}+k_{2}\right)\left((1-m) x+k_{2}\right)}\right) \\
& \left(x-x^{*}\right)\left(y-y^{*}\right) \\
& \leq\left(-b+\frac{c_{1}(1-m)^{2} y^{*}}{\left((1-m) x^{*}+k_{1}\right) k_{1}}\right)\left(x-x^{*}\right)^{2}-\left(\frac{k_{2} c_{2}+(1-m) c_{2} x^{*}}{\left((1-m) x^{*}+k_{2}\right)\left((1-m) x+k_{2}\right)}\right) \\
& \\
& \mu\left(y-y^{*}\right)^{2} \\
& +\left(\frac{-\left(c_{1} k_{1}(1-m)+c_{1}(1-m)^{2} x^{*}\right)}{\left(K+k_{1}\right)^{2}}+\frac{\mu(1-m) c_{2} y^{*}}{k_{2}^{2}}\right)\left(x-x^{*}\right)\left(y-y^{*}\right),
\end{aligned}
$$

and the positive number $K$ indicates the upper boundary to populations of prey from Lemma 2.4.

Choosing the constant

$$
\mu=\frac{c_{1} k_{1}+c_{1}(1-m) x^{*}}{c_{2} y^{*}} \frac{k_{2}^{2}}{\left(K+k_{1}\right)^{2}},
$$

then we obtain the following inequality

$$
\begin{aligned}
\frac{d V}{d t} \leq\left(-b+\frac{c_{1}(1-m)^{2} y^{*}}{\left((1-m) x^{*}+k_{1}\right) k_{1}}\right) & \left(x-x^{*}\right)^{2} \\
& -\left(\frac{k_{2} c_{2}+(1-m) c_{2} x^{*}}{\left((1-m) x^{*}+k_{2}\right)\left((1-m) x+k_{2}\right)}\right) \mu\left(y-y^{*}\right)^{2} .
\end{aligned}
$$

It is obvious that the coefficient of $\left(y-y^{*}\right)^{2}$ is always negative if $k_{1} b>\frac{c_{1}(1-m)^{2} y^{*}}{\left((1-m) x^{*}+k_{1}\right)}$ holds(which leads to $m>1-\frac{b k_{1}}{a_{1}-b x^{*}}$ ), then we have $\frac{d V}{d t}<0$ along all the trajectories in $\mathbb{R}_{+}^{2}$ except $E^{*}$. Thus the function $V(x, y)$ we constructed satisfies all the properties of Lyapunov function.

To determine the qualification of the global stability of system (2.1), we have the following theorem on non existence of limit cycle.

Theorem 2.8. The system does not admit any periodic solution for $m>1-\frac{b k_{1}}{a_{1}}$.

Proof. Let $x(t), y(t)$ be solutions of the system (2.1), then we denote the first equation of the system to be $P(x, y)$ and the second to be $Q(x, y)$ respectively. We define a Dulac function $B(x, y)=\frac{(1-m) x+k_{1}}{x y}$. Calculating the partial derivatives, we obtain 


$$
\begin{array}{rl}
\frac{\partial(B P)}{\partial x}+\frac{\partial(B Q)}{x}=a_{1}(1-m)-b k_{1}-2 & b(1-m) \\
- & {\left[\frac{(1-m) y}{(1-m) x+k_{1}}+\frac{k_{1} y}{x\left[(1-m) x+k_{2}\right]}\right]}
\end{array}
$$

for $x(t), y(t) \in \mathbb{R}_{+}^{2}$, if equation $\frac{\partial(B P)}{\partial x}+\frac{\partial(B Q)}{x} \leq 0$ should be always hold, then it is obvious that $a_{1}(1-m)-b k_{1}<0$, which leads to

$$
m>1-\frac{b k_{1}}{a_{1}} .
$$

Therefore, due to Dulac criterion, system (2.1) has no non-trivial periodic solutions.

Now we can emphasize that, the local stability of the coexist equilibrium $E^{*}\left(x^{*}, y^{*}\right)$ ensures the global asymptotical stability around this point.

\section{ANALYSIS OF THE MODEL WHEN $\tau \neq 0$}

\subsection{Critical value of Hopf bifurcation}

In this section, we concern the dynamic behaviour of the model in the presence of time delay. Particularly, we discuss the stability of the positive equilibrium $E^{*}$ and existence of Hopf bifurcation with time delay $\tau$ as a parameter.

For the purpose, let $X=x-x^{*}, Y=y-y^{*}$, we linearize the system (1.2) about the interior equilibrium solution $E^{*}=\left(x^{*}, y^{*}\right)$ and rewrite the equations to the form(still denote $X$ and $Y$ as $x$ and $y$, respectively):

$$
\left\{\begin{array}{l}
\frac{d x}{d t}=a_{11} x+a_{12} y \\
\frac{d y}{d t}=b_{21} x(t-\tau)+b_{22} y(t-\tau) .
\end{array}\right.
$$

where

$$
\begin{aligned}
a_{11} & =-b x^{*}+\frac{(1-m)^{2} c_{1} x^{*} y^{*}}{\left[(1-m) x^{*}+k_{1}\right]^{2}} ; & a_{12} & =-\frac{c_{1}(1-m) x^{*}}{(1-m) x^{*}+k_{1}} \\
b_{21} & =\frac{(1-m) c_{2} y^{*^{2}}}{\left[(1-m) x^{*}+k_{2}\right]^{2}} ; & b_{22} & =-\frac{c_{2} y^{*}}{(1-m) x^{*}+k_{2}}
\end{aligned}
$$

Then, the Jacobian matrix of system (1.2) for $E^{*}$ is

$$
A=\left(\begin{array}{cc}
a_{11} & a_{12} \\
b_{21} e^{-\lambda \tau} & b_{22} e^{-\lambda \tau}
\end{array}\right) .
$$


By $\operatorname{det}(\lambda I-A)=0$, the characteristic equation corresponding to (3.1) is

$$
\operatorname{det}\left(\begin{array}{cc}
\lambda-a_{11} & -a_{12} \\
-b_{21} e^{-\lambda \tau} & \lambda-b_{22} e^{-\lambda \tau}
\end{array}\right)=0
$$

which leads to the following transcendental equation

$$
\lambda^{2}+A \lambda+(B \lambda+C) e^{-\lambda \tau}=0 .
$$

where $A=-a_{11}, B=-b_{22}, C=a_{11} b_{22}-a_{12} b_{21}$.

If $\tau=0$, then the characteristic equation can be simplify as $\lambda^{2}+(A+B) \lambda+C=0$, which had been studied in previous section. And we have, in the absence of time delay, the system is locally asymptotically stable if $a+b>0$ and $C>0$ hold simultaneously. Now we draw attention to the case of $\tau \neq 0$.

In this condition, we assume that $\lambda(\tau)=\mu(\tau)+i \omega(\tau)$ be an eigenvalue of the system at $E^{*}$. And the stability around the equilibrium will change at $\operatorname{Re}(\lambda)=0$. Therefore, we substituting $\lambda=i \omega$ to the characteristic equation (3.2) and obtain:

$$
-\omega^{2}+A i \omega+i B \omega \cos (\omega \tau)+C \cos (\omega \tau)-i C \sin (\omega \tau)=0 .
$$

Separating the real and imaginary parts, the following equations holds

$$
\left\{\begin{aligned}
-\omega^{2}+B \omega \sin (\omega \tau)+C \cos (\omega \tau) & =0 \\
A \omega+B \omega \cos (\omega \tau)-C \sin (\omega \tau) & =0
\end{aligned}\right.
$$

Since $\sin ^{2} \omega \tau+\cos ^{2} \omega \tau=1$, we get a fourth degree algebraic equation in $\omega$ :

$$
\omega^{4}+\left(A^{2}+B^{2}\right) \omega^{2}-C^{2}=0
$$

The roots of the equation is given by

$$
\omega^{2}=\frac{-\left(A^{2}-B^{2}\right) \pm \sqrt{\left(A^{2}-B^{2}\right)^{2}+4 C^{2}}}{2} .
$$

Thus, the equation just has one unique positive solution, and we define it as $\omega_{0}^{2}$. Substituting $\omega_{0}^{2}$ into (3.3) and solving for $\tau$, we obtain

$$
\tau_{0}=\frac{1}{\omega_{0}} \arctan \left(\frac{A C+B \omega_{0}^{2}}{(C-A B) \omega_{0}}\right)+\frac{2 p \pi}{\omega_{0}}, p=0,1,2 \ldots
$$

Then we need to determine the sign of the derivative of $\operatorname{Re} \lambda(\tau)$ at the point $\tau_{0}$, where $\lambda(\tau)$ is purely imaginary.

Taking the derivative of $\lambda$ with respect to $\tau$ in (3.2), we gain

$$
\left(2 \lambda+A+B e^{-\lambda \tau}\right) \frac{d \lambda}{d \tau}-(B \lambda+C) e^{-\lambda \tau}\left(\tau \frac{d \lambda}{d \tau}+\lambda\right)=0 .
$$


Hence

$$
\frac{d \lambda(\tau)}{d \tau}=\frac{\lambda(B \lambda+C) e^{-\lambda \tau}}{2 \lambda+A+B e^{-\lambda \tau}-\tau(B \lambda+C) e^{-\lambda \tau}}
$$

and from (3.2) we also have

$$
e^{-\lambda \tau}=-\frac{\lambda^{2}+A \lambda}{B \lambda+C}
$$

For convenience, we study $\left(\frac{d \lambda}{d \tau}\right)^{-1}$ instead of $\frac{d \lambda}{d \tau}$, for $\operatorname{sign}\left(\frac{d \operatorname{Re} \lambda}{d \tau}\right)=\operatorname{sign} \operatorname{Re}\left(\frac{d \lambda}{d \tau}\right)^{-1}$.

Thus, we have

$$
\begin{aligned}
\left(\frac{d \lambda}{d \tau}\right)^{-1} & =\frac{2 \lambda+A+B e^{-\lambda \tau}-\tau(B \lambda+C) e^{-\lambda \tau}}{\lambda(B \lambda+C) e^{-\lambda \tau}} \\
& =\frac{B \lambda^{2}+2 C \lambda+A C}{-\lambda\left(\lambda^{2}+A \lambda\right)(B \lambda+C)}-\frac{\tau}{\lambda} .
\end{aligned}
$$

Therefore, substituting $\lambda=i \omega_{0}$ into (3.8) we gain

$$
\begin{aligned}
\operatorname{sign}\left\{\left.\frac{d R e \lambda}{d \tau}\right|_{\lambda=i \omega_{0}}\right\} & =\operatorname{sign}\left\{\left.\operatorname{Re}\left(\frac{d \lambda}{d \tau}\right)^{-1}\right|_{\lambda=i \omega_{0}}\right\} \\
& =\operatorname{sign}\left\{\frac{1}{\omega_{0}^{2}} \frac{-2 C \omega_{0}+\left(A C-\omega_{0}^{2}\right) i}{(-C-A B) \omega_{0}+(A C-B) \omega_{0}^{2} i}\right\} \\
& =\operatorname{sign}\left\{2 C(C+A B) \omega_{0}^{2}+\left(A C-B \omega_{0}^{2}\right)^{2}\right\} \\
& =\operatorname{sign}\left(2 C^{2} \omega_{0}^{2}+A^{2} C^{2}+B^{2} \omega_{0}^{4}\right)=+1 .
\end{aligned}
$$

Which implies that in the case above, there exist only one imaginary root $\lambda=i \omega_{0}$. Thus only one crossing of imaginary axis is from left to right as $\tau$ increase, and the stability of trivial solution can only be lost and not regained. The zero solution is asymptotically stable for $\tau=0$, then it is asymptotically stable for $\tau<\tau_{0}$, and becomes unstable for $\tau>\tau_{0}$. Summarize the discussion above, we have following theorem:

Theorem 3.1. Under the condition that the interior equilibrium $E^{*}$ is asymptotically stable for non-delay system,

(i) for system (1.2), its interior equilibrium is asymptotically stable for $\tau \in\left[0, \tau_{0}\right)$;

(ii) system (1.2) undergoes a Hopf bifurcation at the origin when $\tau=\tau_{0}$. That is, the delayed system (1.2) has a branch of periodic solutions bifurcating from the interior equilibrium near the critical value $\tau=\tau_{0}$. 


\subsection{Direction and stability of Hopf bifurcation}

In previous section, we have investigated that system (1.2) undergoes Hopf bifurcations at delay $\tau_{0}$, and indicate the existence of the periodic solutions in the neighbourhood of the interior equilibrium. In this section, we shall determine the direction of the Hopf bifurcation and stability of the bifurcating periodic solutions of the system at $\tau=\tau_{0}$ by applying the normal theory and the center manifold theorem due to the methods of Kuznetsov [16].

Now we rewrite the system (1.2) as the form of

$$
\frac{d X}{d t}=A X+F(X)
$$

where $X=(x, y)$ and $A, F$ respectively be the liner and nonlinear term of the Tailor's series expansion to the right hand side of system.

In this regard, let $\xi_{1}=x-x^{*}, \xi_{2}=y-y^{*}$, where $x^{*}, y^{*}$ are coordinates of the equilibrium to the model we considered. We translate the origin of the coordinates to the equilibrium, then the equations can be written as the form into

$$
\left\{\begin{array}{l}
\dot{\xi}_{1}=a_{11} \xi_{1}+a_{12} \xi_{2}+a_{13} \xi_{1}^{2}+a_{14} \xi_{1} \xi_{2}+a_{15} \xi_{1}^{3}=F_{1}\left(\xi_{1}, \xi_{2}\right) \\
\dot{\xi}_{2}=b_{21} \xi_{1}(t-\tau)+b_{22} \xi_{2}(t-\tau)+b_{23} \xi_{1}^{2}(t-\tau)+b_{24} \xi_{1}(t-\tau) \xi_{2} \\
\quad+b_{25} \xi_{2}(t-\tau) \xi_{1}+b_{26} \xi_{1}^{2}(t-\tau) \xi_{2}=F_{2}\left(\xi_{1}, \xi_{2}\right)
\end{array}\right.
$$

where $a_{11}, \ldots, a_{15}, b_{21}, \ldots, b_{26}$ are coefficient as follow:

$$
\begin{aligned}
& a_{11}=-b x^{*}+\frac{(1-m)^{2} c_{1} x^{*} y^{*}}{\left[(1-m) x^{*}+k_{1}\right]^{2}} \\
& a_{12}=-\frac{(1-m) c_{1} x^{*}}{(1-m) x^{*}+k_{1}} ; \\
& a_{13}=-b x^{*}+\frac{(1-m)^{2} c_{1} y^{*}}{\left[(1-m) x^{*}+k_{1}\right]^{2}}-\frac{(1-m)^{3} c_{1} x^{*} y^{*}\left[(1-m) x^{*}+k_{1}\right]}{\left[(1-m) x^{*}+k_{1}\right]^{4}} ; \\
& a_{14}=-\frac{c_{1}(1-m)}{(1-m) x^{*}+k_{1}} ; \\
& a_{15}=-\frac{(1-m)^{3} c_{1} y^{*}\left[(1-m) x^{*}+k_{1}\right]}{\left[(1-m) x^{*}+k_{1}\right]^{4}}
\end{aligned}
$$

and

$$
\begin{array}{lc}
b_{21}=\frac{(1-m) c_{2} y^{*^{2}}}{\left[(1-m) x^{*}+k_{2}\right]^{2}} ; & b_{22}=-\frac{c_{2} y^{*}}{(1-m) x^{*}+k_{2}} \\
b_{23}=-\frac{(1-m)^{2} c_{2} y^{*^{2}}\left[(1-m) x^{*}+k_{2}\right]}{\left[(1-m) x^{*}+k_{2}\right]^{4}} ; & b_{24}=\frac{(1-m) c_{2} y^{*}}{\left[(1-m) x^{*}+k_{2}\right]^{2}} \\
b_{25}=-\frac{c_{2}}{(1-m) x^{*}+k_{2}} ; & b_{26}=-\frac{(1-m)^{2} c_{2} y^{*}\left[(1-m) x^{*}+k_{2}\right]}{\left[(1-m) x^{*}+k_{2}\right]^{4}}
\end{array}
$$


Thus, the system shall be represented as

$$
\dot{\xi}=A \xi+\frac{1}{2} B(\xi, \xi)+\frac{1}{6} C(\xi, \xi, \xi)+O\left(\|\xi\|^{4}\right),
$$

where $\xi=\left(\xi_{1}, \xi_{2}\right)^{T}$ and the matrix $A\left(\omega_{0} \tau_{0}\right)$ takes the form of

$$
A=\left(\begin{array}{cc}
a_{11} & a_{12} \\
b_{21} e^{-i \omega_{0} \tau_{0}} & b_{22} e^{-i \omega_{0} \tau_{0}}
\end{array}\right) .
$$

Due to the formula from Kuznetsov that the multilinear functions $B$ and $C$ can be calculated as follow:

$$
B_{i}(x, y)=\left.\sum_{j, k=1}^{2} \frac{\partial^{2} F_{i}\left(\xi_{1}, \xi_{2}\right)}{\partial \xi_{j} \partial \xi_{k}}\right|_{\omega_{0} \tau_{0}} x_{j} y_{k}, i=1,2
$$

and

$$
C_{i}(x, y, z)=\left.\sum_{j, k, l=1}^{2} \frac{\partial^{3} F_{i}\left(\xi_{1}, \xi_{2}\right)}{\partial \xi_{j} \partial \xi_{k} \partial \xi_{l}}\right|_{\omega_{0} \tau_{0}} x_{j} y_{k} z_{l}, i=1,2 .
$$

Now using the formula above, we can easily get the function $B, C$ take on the planar vectors $\xi=\left(\xi_{1}, \xi_{2}\right)^{T}, \eta=\left(\eta_{1}, \eta_{2}\right)^{T}, \zeta=\left(\zeta_{1}, \zeta_{2}\right)^{T}$ the values

$$
B(\xi, \eta)=\left(\begin{array}{c}
2 a_{13} \xi_{1} \eta_{1}+a_{14}\left(\xi_{1} \eta_{2}+\xi_{2} \eta_{1}\right) \\
2 b_{23} e^{-2 i \omega_{0} \tau_{0}} \xi_{1} \eta_{1}+b_{24} e^{-i \omega_{0} \tau_{0}}\left(\xi_{1} \eta_{2}+\xi_{2} \eta_{1}\right)+b_{25} e^{-i \omega_{0} \tau_{0}} \xi_{2} \eta_{2}
\end{array}\right),
$$

and in the same way we have

$$
C(\xi, \eta, \zeta)=\left(\begin{array}{c}
6 a_{15} \xi_{1} \eta_{1} \zeta_{1} \\
2 b_{26} e^{-2 i \omega_{0} \tau_{0}} \xi_{1} \eta_{1} \zeta_{2}
\end{array}\right)
$$

Next we determine the eigenvector $q$ of $A$ associated to the eigenvalue $i \omega_{0} \tau_{0}$ and the eigenvector $p$ of $A^{T}$ associated to the eigenvalue $-i \omega_{0} \tau_{0}$.

From the discussion above, we have

$$
A q=i \omega_{0} \tau_{0} q
$$

Let the vector $q$ takes the form of $(1, \rho)^{T}$ i.e.,

$$
\left(\begin{array}{cc}
i \omega_{0} \tau_{0}-a_{11} & -a_{12} \\
-b_{21} e^{-i \omega_{0} \tau_{0}} & i \omega_{0} \tau_{0}-b_{22} e^{-i \omega_{0} \tau_{0}}
\end{array}\right)\left(\begin{array}{l}
1 \\
\rho
\end{array}\right)=\left(\begin{array}{l}
0 \\
0
\end{array}\right)
$$

then we get $\rho=-\frac{a_{11}-i \omega_{0} \tau_{0}}{a_{12}}$ thus we have

$$
q \sim\left(\begin{array}{c}
1 \\
-\frac{a_{11}-i \omega_{0} \tau_{0}}{a_{12}}
\end{array}\right) .
$$


Similarly, from that $p$ is the eigenvector of $A^{T}$ we obtain

$$
A^{T} p=-i \omega_{0} \tau_{0} p
$$

let vector $p$ takes the form of $(1, \sigma)^{T}$

i.e.,

$$
\left(\begin{array}{cc}
a_{11}+i \omega_{0} \tau_{0} & b_{21} e^{-i \omega_{0} \tau_{0}} \\
a_{12} & b_{22} e^{-i \omega_{0} \tau_{0}}+i \omega_{0} \tau_{0}
\end{array}\right)\left(\begin{array}{l}
1 \\
\sigma
\end{array}\right)=\left(\begin{array}{l}
0 \\
0
\end{array}\right)
$$

Hence we have $\sigma=-\frac{\left(a_{11}+i \omega_{0} \tau_{0}\right) e^{i \omega_{0} \tau_{0}}}{b_{21}}$,

$$
p \sim\left(\begin{array}{c}
1 \\
-\frac{\left(a_{11}+i \omega_{0} \tau_{0}\right) e^{i \omega_{0} \tau_{0}}}{b_{21}}
\end{array}\right) .
$$

By the necessary normalization $\langle p, q\rangle=1$, where $\langle p, q\rangle=\overline{p_{1}} q_{1}+\overline{p_{2}} q_{2}$ so we take

$$
k=\frac{a_{12} b_{21}}{a_{12} b_{21}+\left[a_{11}^{2}-\omega_{0}^{2} \tau_{0}^{2}-2 a_{11} \omega_{0} \tau_{0} i\right] e^{i \omega_{0} \tau_{0}}},
$$

then, $p, q$ are proper eigenvectors we need, where

$$
p=k\left(\begin{array}{c}
1 \\
-\frac{a_{11}+i \omega_{0} \tau_{0} e^{i \omega_{0} \tau_{0}}}{b_{21}}
\end{array}\right) ; \quad q=\left(\begin{array}{c}
1 \\
-\frac{a_{11}-i \omega_{0} \tau_{0}}{a_{12}}
\end{array}\right) .
$$

Then, any vector $\xi \in \mathbb{R}^{2}$ can be uniquely represented for any small $\tau$ as $\xi=z q+\overline{z q}$. Let $z(t)=\langle p, \xi\rangle$, for

$$
\langle p, \bar{q}\rangle=\left\langle p, \frac{1}{\bar{\lambda}} A \bar{q}\right\rangle=\frac{1}{\bar{\lambda}}\langle p, \bar{\lambda} \bar{q}\rangle=\frac{1}{\bar{\lambda}}\langle\bar{\lambda} p, \bar{q}\rangle=\frac{1}{\bar{\lambda}}\left\langle A^{T} p, \bar{q}\right\rangle=\frac{1}{\bar{\lambda}}\langle p, \bar{q}\rangle .
$$

We have

$$
\left(1-\frac{\lambda}{\bar{\lambda}}\right)\langle p, \bar{q}\rangle=0
$$

But $\lambda \neq \bar{\lambda}$ because for all sufficient small $|z|$ we have $\omega(\tau) \neq 0$, thus, the only possibility is $\langle p, \bar{q}\rangle=0$. Hence, complex variable $z$ obviously satisfies

$$
\begin{aligned}
\dot{z}(t)=\langle p, \dot{\xi}\rangle=\langle p, A(\xi)+F(\xi)\rangle=\langle p, A(z q+\overline{z q})\rangle+\langle p, A(z q+\overline{z q})\rangle & \\
& =i \omega_{0} \tau_{0} z+\langle p, A(z q+\overline{z q})\rangle
\end{aligned}
$$

We rewrite this equation as

$$
\dot{z}(t)=i \omega_{0} \tau_{0} z+g(z, \bar{z}),
$$

where

$$
g(z, \bar{z})=\langle p, A(z q+\overline{z q})\rangle
$$




$$
=g_{20} \frac{z^{2}}{2}+g_{11} z \bar{z}+g_{02} \frac{\bar{z}^{2}}{2}+g_{21} \frac{z^{2} \bar{z}}{2}+\cdots .
$$

Now use the Lemma 3.6 from [16], we make an invertible parameter-dependent change of complex coordinate

$$
z=w+\frac{h_{20}}{2} w^{2}+h_{11} w \bar{w}+\frac{h_{02}}{2} \bar{w}^{2}+\frac{h_{30}}{6} w^{3}+\frac{h_{12}}{2} w \bar{w}^{2}+\frac{h_{03}}{6} \bar{w}^{3} .
$$

Then, for all sufficiently small $\tau$, equation (3.10) can be simplify into the form of with only the resonant cubic term:

$$
\dot{w}=i \omega_{0} \tau_{0} w+c_{1}(0) w^{2} \bar{w}+O\left(|w|^{4}\right) .
$$

Comparing the coefficients of the quadratic terms in expressions for $\dot{z}$ in front of the $w|w|^{2}$-term leads to

$$
c_{1}(0)=\frac{i}{2 \tau_{0} \omega_{0}}\left(g_{20} g_{11}-2\left|g_{11}\right|^{2}-\frac{1}{3}\left|g_{02}\right|^{2}\right)+\frac{g_{21}}{2} .
$$

Next,comparing the quadratic and cubic terms of Taylor series in $g(z, \bar{z})$,for

$$
B(z q+\overline{z q}, z q+\overline{z q})=z^{2} B(q, q)+2 z \bar{z} B(q, \bar{q})+\bar{z}^{2} B(\bar{q}, \bar{q})
$$

we can compute the coefficients by formula below:

$$
g_{20}=\langle p, B(q, q)\rangle, \quad g_{11}=\langle p, B(q, \bar{q})\rangle, \quad g_{02}=\langle p, B(\bar{q}, \bar{q})\rangle,
$$

and by the same way of calculations to $C$ we have

$$
g_{21}=\langle p, C(q, q, \bar{q})\rangle
$$

Using them we get the following quantities

$$
c_{1}(0)=\frac{i}{2 \tau_{0} \omega_{0}}\left(g_{20} g_{11}-2\left|g_{11}\right|^{2}-\frac{1}{3}\left|g_{02}\right|^{2}\right)+\frac{g_{21}}{2},
$$

from the expression of $c_{1}(0)$, it is easy to compute the value of $\mu_{2}, \beta_{2}$ and $t_{2}$ as follow:

$$
\begin{aligned}
& \mu_{2}=-\frac{\operatorname{Re}\left\{c_{1}(0)\right\}}{\operatorname{Re}\left\{\lambda^{\prime}\left(\tau_{0}\right)\right\}}, \\
& \beta_{2}=2 \operatorname{Re}\left\{c_{1}(0)\right\}, \\
& t_{2}=-\frac{\operatorname{Im}\left\{c_{1}(0)\right\}+\mu_{2} \operatorname{Im}\left\{\lambda^{\prime}\left(\tau_{0}\right)\right\}}{\tau_{0} \omega_{0}} .
\end{aligned}
$$

Then, due to Hassard's book [10], we state the following theorem: 
Theorem 3.2. $\quad$ 1. $\mu=0$ is Hopf bifurcation value of the system;

2. The sign of $\mu_{2}$ determined the direction of Hopf bifurcation:if $\mu_{2}>0\left(\mu_{2}<\right.$ $0)$, then the Hopf bifurcation is supercritical(subcritical) and the bifurcating periodic solution exist for $\tau>\tau_{0}\left(\tau<\tau_{0}\right)$;

3. $\beta_{2}$ determines the stability of the bifurcating periodic solution:the bifurcating periodic solution is stable (unstable) if $\beta_{2}<0\left(\beta_{2}>0\right)$;

4. $t_{2}$ determines the period of the bifurcating periodic solution:the period increase (decrease) if $t_{2}>0\left(t_{2}<0\right)$.

\section{NUMERICAL ANALYSIS AND SIMULATIONS}

In this section, we perform numerical computations to validate our analytical findings and stability results obtained in previous section. We show the dynamic property of the system by using different sets of parametric values in both delayed and non-delayed system. Corresponding to the non-delayed system (2.1), we consider the following two sets of parametric values:

$$
a_{1}=2, \quad b=0.023, \quad c_{1}=5, \quad k_{1}=60, \quad a_{2}=0.5, \quad c_{2}=0.5, \quad k_{2}=40,
$$

and

$$
a_{1}=2, \quad b=0.002, \quad c_{1}=5, \quad k_{1}=60, \quad a_{2}=0.5, \quad c_{2}=0.5, \quad k_{2}=40 .
$$

For the parametric values given in (4.1), we firstly consider the existence of the interior equilibrium with respect to refuge parameter $m$ and choose $m=0.5$, then these lead $x^{*}=11.3595, y^{*}=45.6798$ and the interior equilibrium clearly exist under the condition that $m>1-\frac{a_{1} c_{2} k_{1}}{a_{2} c_{1} k_{2}}=0.4$. For this set of parameter values with $m=0.5$, it is obvious that the interior equilibrium is also global asymptotically stable for $m>1-\frac{b k_{1}}{a_{1}}=0.31$ in which it must be hold that the sufficient condition of asymptotically stability is satisfied. Fig(1) depicts clearly the global stability and existence of the interior equilibrium $E^{*}\left(x^{*}, y^{*}\right)$ under the constructed parameter sets (4.1) with $m=0.5$. Fig(2) shows when we choose $m=0.4$ under parameter set (4.1), there is no coexist equilibrium of system $(2.1)$ and $E_{2}=(0,40)$ becomes an asymptotically stable point.

Then we illustrate the behaviour of the model with respect to the existence of limit cycle. Consider the values of parameter set (4.2) where $a_{1}=2, b=0.002, c_{1}=$ $5, k_{1}=60, a_{2}=0.5, c_{2}=0.5$ and $k_{2}=40$ with $m=0.5$. Numerical computation shows that the interior equilibrium $E^{*}$ exist but it may not be global stable for 


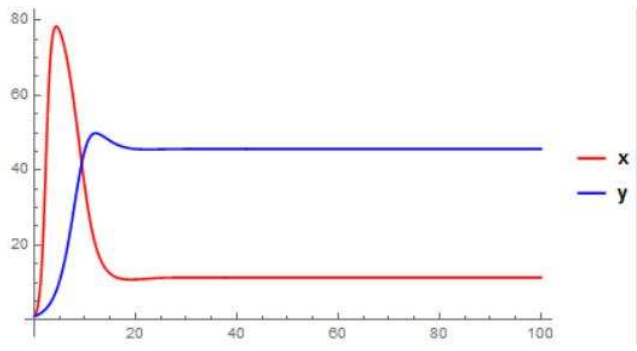

(a)

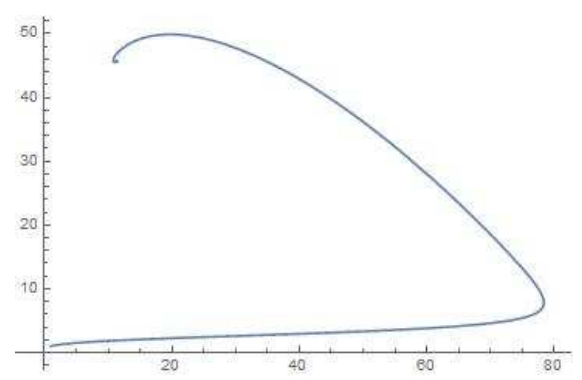

(b)

Figure 1: Simulating solution of system (3) for parameter set (19) with $\mathrm{m}=0.5$ and initial values $(1,1)$. (a) The trajectories of prey and predator densities versus time. (b) The phase portrait shows the stability of $E^{*}$ for given parameters.

Table 1: The equilibria of the non-delay system with respect to the parameter $\mathrm{m}$.

\begin{tabular}{|c|c|c|c|}
\hline Parameters & $E_{1}$ & $E_{2}$ & $E^{*}$ \\
\hline$m<1-\frac{a_{1} c_{2} k_{1}}{a_{2} c_{1} k_{2}}$ & unstable & stable & does not exist \\
$m>1-\frac{a_{1} c_{2} k_{1}}{a_{2} c_{1} k_{2}}$ & unstable & unstable & exist \\
$m>1-\frac{b k_{1}}{a_{1}}$ & unstable & unstable & asymptotic stable \\
\hline
\end{tabular}

$m<1-\frac{b k_{1}}{a_{1}}=0.94$ and $m>1-\frac{a_{1} c_{2} k_{1}}{a_{2} c_{1} k_{2}}=0.4$. Fig(3) shows there is a periodic solution around the equilibrium and the solution oscillates with initial values $(1,1)$.

Next we consider the system incorporating time delays. We make the first delayed set of parametric values based on the set (19) constructed previous, which we add delay $\tau=1.9$ in to it: $a_{1}=2, b=0.023, c_{1}=5, k_{1}=60, a_{2}=0.5, c_{2}=0.5, k_{2}=40$ and $\tau=1.9$. From the calculation before we know that the positive equilibrium $E^{*}(11.3595,45.6798)$ is global asymptotically stable when $m>0.4$. This time we still choose $m=0.5$ to investigate the effort of delay and compute from (13), we have the critical value of delay is $\tau_{0}=2.1895$. Fig(4) shows that for the given parametric values (19) and $m=0.5$, the system is still asymptotically stable around $E^{*}(11.3595,45.6798)$ but with a clear oscillation than that of non-delayed case, for $\tau=1.9<\tau_{0}$.

From computing the critical value of the Hopf bifurcation, we then construct another parameter set: $a_{1}=2, b=0.023, c_{1}=5, k_{1}=60, a_{2}=0.5, c_{2}=0.5, k_{2}=$ 


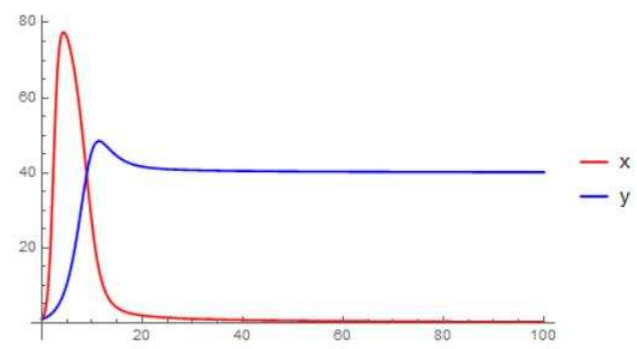

(a)

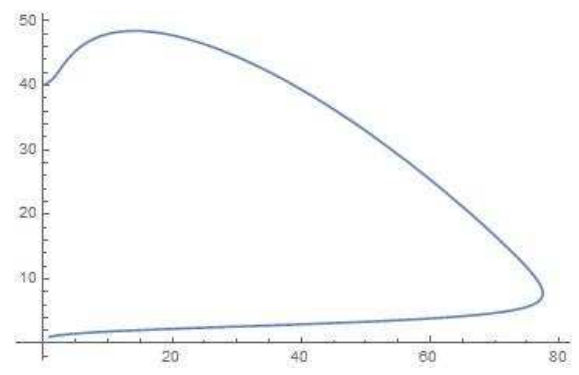

(b)

Figure 2: Simulating solution of system (3) for parameter set (19) with $\mathrm{m}=0.4$ and initial values $(1,1)$. (a) The trajectories of prey and predator densities versus time. (b) The phase portrait shows the stability of $E_{2}$ for given parameters.

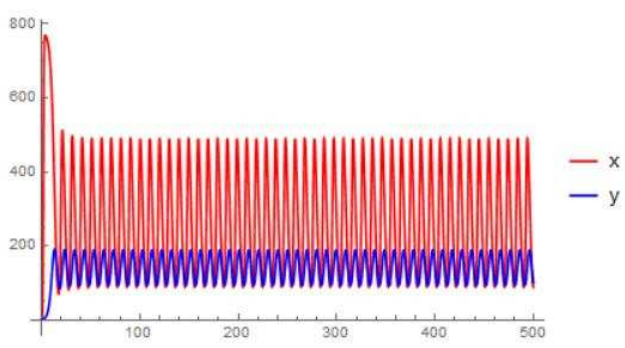

(a)

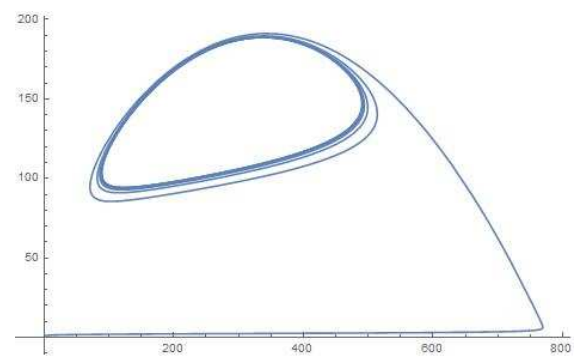

(b)

Figure 3: Simulating solution of system (3) for parameter set (19) with $\mathrm{m}=0.5$ and initial values $(1,1)$. (a) The trajectories of prey and predator densities versus time. (b) The phase portrait shows the periodic solution corresponding to set $(20)$ around the coexist equilibrium $E_{*}$.

40, $m=0.5$ and $\tau=2.2$. The Fig(5) shows that for a more bigger time delay $\tau=2.2>\tau_{0}$, the interior equilibrium lost the stability and the system exhibit a periodic solution around $E^{*}$.

For the delayed system we make the effort of prey refuge more powerful and construct a new set of parameters: $a_{1}=2, b=0.023, c_{1}=5, k_{1}=60, a_{2}=0.5, c_{2}=$ $0.5, k_{2}=40, m=0.8$ and $\tau=2.2$. $\operatorname{Fig}(6)$ show that for a delayed system the parameter $m$ has a stabilize effort on the system. The limit cycle degenerates and the model again becomes stable around to its equilibrium solution $E^{*}(55.7015,51.1403)$. 


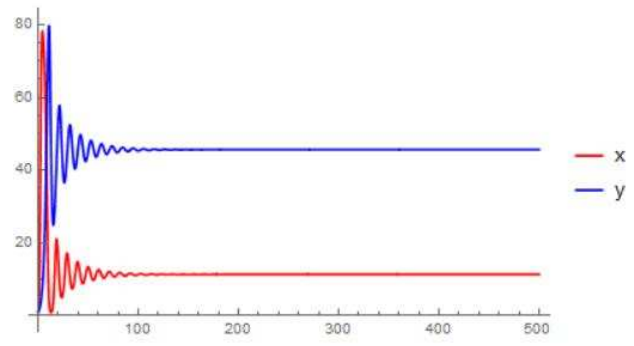

(a)

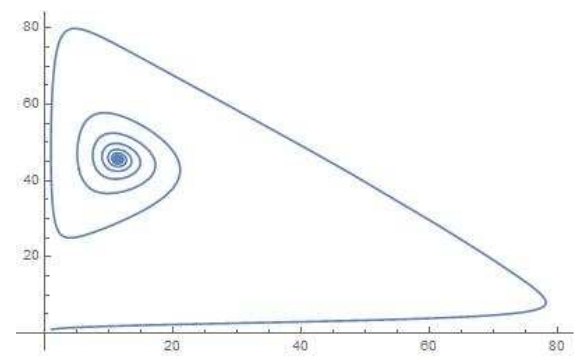

(b)

Figure 4: Simulating solution of system (2) for $\mathrm{m}=0.5$ and $\tau=1.9$ : $E^{*}$ is still asymptotically stable for $\tau<\tau_{0}=2.1895$. (a) The trajectories of prey and predator densities versus time with initial values $(1,1)$. (b) The phase portrait.

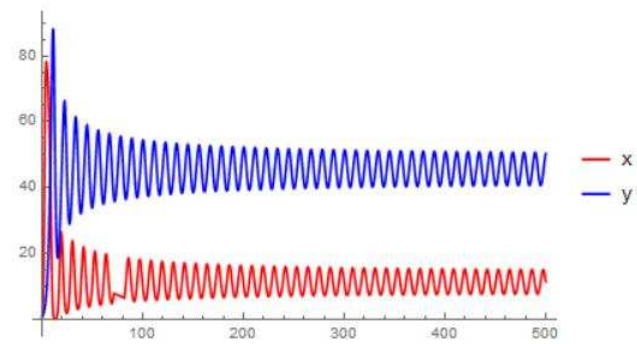

(a)

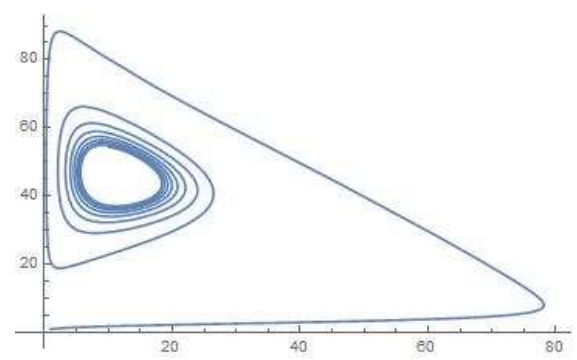

(b)

Figure 5: Simulating solution of system (2) for $\mathrm{m}=0.5$ and $\tau=2.2$ : there is a stable periodic solutions near $E^{*}$. (a) The trajectories of prey and predator densities versus time with initial values $(1,1)$. (b) The phase portrait.

\section{CONCLUDING REMARKS}

For more species are currently threatened with extinction and ecological system becomes fragile and nearly collapse in some regions due to deforest or environmental pollution, sustainable development of ecosystem and species conservation come to be a more attracting field of ecology research. That motivates the establishment of some protected areas for species. The establishment of reserve for species in danger may decrease the interactions among them, which makes a small proportion of prey species could survive from predation for refuge available.

Based on this idea, our present paper deals with a prey-predator interacting system incorporating a refuge to prey. For non-delayed model we analysis the local stability 


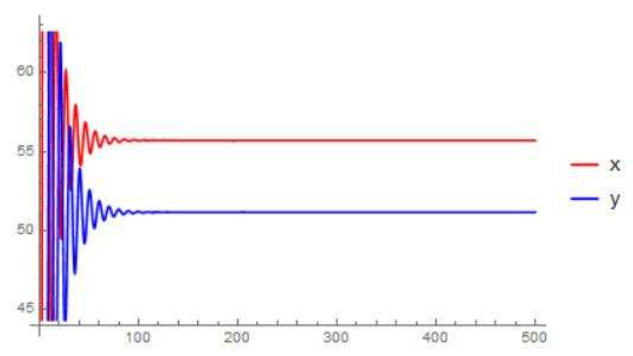

(a)

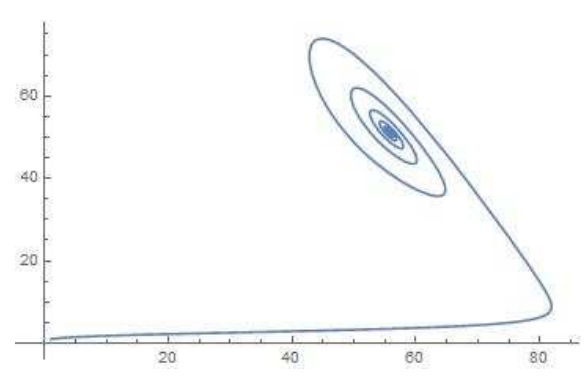

(b)

Figure 6: Simulating solution of system (2) for $\mathrm{m}=0.8$ and $\tau=2.2$ : $E^{*}$ becomes asymptotically stable with a more bigger $m$ for its stabilize effort to system. (a) The trajectories of prey and predator densities versus time with initial values $(1,1)$. (b) The phase portrait.

of every equilibria and show the fact that prey reserve plays no role in determining the stability of the trivial solution $E_{0}$ and $E_{1}$, but a positive role in the case of stability of coexist solution $E^{*}$. We proved that the existence of $E^{*}$ is contradict to the stability of $E_{2}$, and prey reserve has positive influence on the global asymptotically stable of the interior equilibrium as $m>1-\frac{b k_{1}}{a_{1}}$. And the numerical simulation verifies the existence of periodic solution when the refuge can reserve enough number of prey.

The time delay is considered in the predators function to represent its gestation delay for making the model more realistic. The interior equilibrium of non-delay system is asymptotically stable under some parametric restrictions and the system experiences Hopf-bifurcation when the delay parameters $\tau$ attains a particular critical value $\tau_{0}$. When the time delay does not big enough, the populations of both species oscillate in finite time. But in the case of the system with a long time delay, the equilibrium will lost its stability and a limit cycle emerge. Then we show that the parameter of refuge $m$ also has a stabilizing effect to delayed system.

\section{ACKNOWLEDGMENTS}

This work was supported by the National Nature Science Foundation of China (Grant No. 41571016).

\section{REFERENCES}

[1] N. Ali, M. Jazar, Global dynamics of a modified Leslie-Gower predator-prey model with Crowley-Martin functional responses, Journal of Applied Mathematics and 
Computing, 43(1-2) (2013), 271-293

[2] M. A. Aziz-Alaoui, Study of a Leslie-Gower-type tritrophic population model, Chaos, Solitons and Fractals, 14(8) (2002), 1275-1293

[3] M. A. Aziz-Alaoui, M. D. Okiye, Boundedness and global stability for a predatorprey model with modified Leslie-Gower and Holling-type II schemes, Applied Mathematics and Computation, 217(22) (2011), 9085-9104

[4] A. Berryman, The Orgins and Evolution of Predator-Prey Theory, Ecology, 73(5) (1992), 1530-1535.

[5] P. A. Braza, Predator-prey dynamics with square root functional responses, Nonlinear Analysis Real World Applications, 13(4) (2012), 1837-1843

[6] F. D. Chen, On a nonlinear non-autonomous predator-prey model with diffusion and distributed delay, J. Comput. Appl. Math, 180(1) (2005), 33-49

[7] H. I. Freedman, Deterministic Mathematical Models in Population Ecology, Marcel Dekker, 1980

[8] G. F. Gause, Experimental studies on the struggle for existence, Journal of Experimental Biology, 9 (1932), 389-402

[9] I. Hanski, L. Hansson, H. Henttonen, Specialist Predators, Generalist Predators, and the Microtine Rodent Cycle, Journal of Animal Ecology, 60(1) (1991), 353367

[10] B. D. Hassard, N. D. Kazarinoff, Y. H. Wan, Theory and Application of Hopf Bifurcation, Cambridge University Press, 1981

[11] C. Holling, The functional response of predators to prey density and its role in mimicry and population regulations, Memoirs of the Entomological Society Canada, 97(45) (1965), 3-60

[12] S. Jana, M. Chakraborty, K. Chakraborty, T. K. Kar, Global stability and bifurcation of time delayed prey-predator system incorporating prey refuge, Mathematics and Computers in Simulation, 85(3) (2012), 57-77

[13] T. K. Kar, A. Ghorai, Dynamic behaviour of a delayed predator-prey model with harvesting, Applied Mathematics and Computation, 217(22) (2011), 9085-9104

[14] M. Kot, Elements of mathematical ecology, Cambridge University Press, 2001

[15] Y. Kuang, Delay Differential Equations with Applications in Population Dynamics, Academic Press, 1993

[16] Y. A. Kuznetsov, Elements of Applied Bifurcation Theory, Springer, 1997

[17] P. H. Leslie, Some further notes on the use of matrices in population mathematics, Biometrika, 35(3/4) (1948), 213-245 
[18] P. H. Leslie, J. C. Gower, The Properties of a Stochastic Model for the PredatorPrey Type of Interaction Between Two Species, Biometrika, 47(3/4) (1960), 219 234

[19] Y. Li, C. Li, Stability and Hopf bifurcation analysis on a delayed Leslie-Gower predatorCprey system incorporating a prey refuge, Applied Mathematics and Computation, 219(9) (2013), 4576-4589

[20] X. Liu, M. Han, Chaos and Hopf bifurcation analysis for a two species predatorprey system with prey refuge and diffusion, Nonlinear Analysis Real World Applications, 12(2) (2011), 1047-1061

[21] A. F. Nindjin, M. A. Aziz-Alaoui, M. Cadivel, Analysis of a predator-prey model with modified Leslie-Gower and Holling-type II schemes with time delay, Nonlinear Analysis Real World Applications, 7(5) (2006), 1104-1118

[22] E. C. Pielou, An Introduction to Mathematical Ecology, Wiley-Interscience, 1969

[23] J. P. Tripathi, S. Abbas, M. Thakur, A density dependent delayed predator-prey model with Beddington-DeAngelis type function response incorporating a prey refuge, Communications in Nonlinear Science and Numerical Simulation, 22(1-3) (2015), 427-450

[24] R. Yuan, W. Jiang, Y. W, Saddle-node-Hopf bifurcation in a modified LeslieGower predator-prey model with time-delay and prey harvesting, Journal of Mathematical Analysis and Applications, 422(2) (2015), 1072-1090 
